\title{
THE DISCRETE COUNTABLE CHAIN CONDITION
}

\section{MARGARET REAMES WISCAMB}

I. Introduction. In this paper, we investigate a topological property, the discrete countable chain condition (DCCC), which is a generalization of separability, of the Lindelöf property, and of countable compactness. These relationships are studied in $\$ 2$ and this property is shown to be equivalent to an apparently weaker condition (Theorem 2.6).

A topological space $R$ is said to satisfy the countable chain condition (CCC) if every collection of disjoint open sets is countable.

A topological space $R$ is said to satisfy the discrete countable chain condition (DCCC) if every discrete collection of open sets is countable.

It is well known that a separable space satisfies the countable chain condition, and it is easy to find an example to show that the converse fails $[6$, p. 60]. That CCC implies DCCC is obvious from the definitions.

An example of E. Michael [7] gives us a topological space that satisfies the DCCC but not the CCC. This space is Lindelöf, hence satisfies the DCCC (Theorem 2.1), but has an uncountable subset with the discrete topology, hence does not satisfy the CCC.

II. Relations between the DCCC and other topological properties. There are examples of Lindelöf spaces which do not satisfy the CCC and spaces satisfying the CCC which are not Lindelöf, however, Theorem 2.1 gives us a relation between a Lindelöf space and a space satisfying the DCCC.

For convenience in stating the first few theorems, we label three conditions as follows:

(i) $R$ is Lindelöf.

(ii) Every uncountable subset of $R$ has at least one cluster point.

(iii) $R$ satisfies the DCCC.

Theorem 2.1. For a $T_{1}$ space $R$, (i) implies (ii) and (ii) implies (iii).

Proof. (i) implies (ii). Suppose $S$ is an uncountable subset of $R$ and $S$ has no cluster point in $R$. Then for each $p \in S$ there exists a neighborhood $U(p)$ which contains no other element of $S$. Moreover, $S$ is closed since it has no cluster points. Then $\{R-S, U(p) \mid p \in S\}$ is an uncountable open covering of $R$ which has no countable subcovering.

Received by the editors February 27, 1967. 
(ii) implies (iii) is easy.

Since a $T_{1}$ space is countably compact if every infinite set has a cluster point, we have the following corollary:

Corollary 2.2. A countably compact space satisfies the DCCC.

The converse of this is not true. The real line with the usual topology satisfies (ii), hence the DCCC, but is not countably compact.

THEOREM 2.3. A regular space $R$ is Lindelöf if and only if it is paracompact and satisfies the DCCC.

This result follows from a theorem of E. Michael [8]. A regular topological space is paracompact if and only if every open covering has a $\sigma$-discrete open refinement.

We also note that a metric space satisfying the DCCC is second countable. This follows at once from Bing's [3] characterization of a metrizable space; a regular topological space $R$ is metrizable if and only if it has a $\sigma$-discrete open basis.

It is not true in general that (ii) implies (i). The set of ordinals less than the first uncountable ordinal with the interval topology is countably compact but not Lindelöf.

A space is pointwise paracompact or metacompact if every open covering has a point-finite open refinement.

Theorem 2.4. If $R$ is metacompact, then (ii) implies (i).

Proof. The proof of this is a trivial modification of a proof of Arens and Dugundji [1].

It is not true that (iii) implies (ii). To see this, we first observe that any closed subspace of a topological space satisfying (ii) also enjoys (ii).

Exercise $1 \mathrm{~L}$ from Kelley [6, p. 59] is a space which is separable, hence satisfies the DCCC. It has a closed subspace which does not satisfy (ii).

A topological space $R$ is collectionwise normal if for every discrete collection of sets $\left\{A_{\alpha} \mid \alpha \in Q\right\}$ there exists a disjoint collection $\left\{U_{\alpha} \mid \alpha \in a\right\}$ of open subsets of $R$ such that $A_{\alpha} \subset U_{\alpha}$ for every $\alpha \in Q$. Dowker has proved [5] that if $\left\{V_{\lambda} \mid \lambda \in \Lambda\right\}$ is a disjoint collection of open sets of a normal space $R$, if $D_{\lambda} \subset V_{\lambda}$ for each $\lambda$, and if $U\left\{D_{\lambda} \mid \lambda \in \Lambda\right\}$ is closed, then there exists a discrete family $\left\{W_{\lambda} \mid \lambda \in \Lambda\right\}$ of open subsets of $R$ such that $D_{\lambda} \subset W_{\lambda} \subset V_{\lambda}$ for all $\lambda$.

THEOREM 2.5. If $R$ is collectionwise normal, then (iii) implies (ii).

Proof. Suppose $S$ is an uncountable infinite subset of $R$ having 
no cluster point in $R$. Then $S$ is a discrete collection of points, and there exists a disjoint collection of open sets $\{U(p) \mid p \in S\}$ where $p \in U(p)$. Since $S$ is closed and $R$ is normal, by Dowker's theorem we can find a discrete family of open sets $\{W(p) \mid p \in S\}$ such that $p \in W(p) \subset U(p)$ for every $p \in S$. Thus $\{W(p) \mid p \in S\}$ is an uncountable discrete collection of open sets, and $R$ does not satisfy the DCCC.

THeOREM 2.6. If $R$ is a regular space, then $R$ satisfies the DCCC if and only if every locally finite collection of open sets of $R$ is countable.

Proof. Sufficiency is obvious. Therefore, let $u$ be a locally finite collection of open sets in $R$. We assume $R$ is well ordered, and for $p \in R$, we put $\mathcal{u}(p)=\{U \mid U \in \mathcal{U} ; p$ is the first element in $U\}$.

By the local finiteness of $u, \mathcal{u}(p)$ is a finite set which may be empty.

Each set in $\mathcal{U}$ is contained in one and only one $\mathcal{u}(p)$ and

$$
q<p \text { implies } q \notin U \text { for } U \in \mathcal{u}(p) \text {. }
$$

We assert that the collection $\{u(p) \mid u(p) \neq \varnothing, p \in R\}$ is countable. To see this, choose one set $U(p)$ from each nonempty $u(p)$. Then the collection $\{U(p)\}$ is locally finite since $\mathfrak{u}$ is.

Since $R$ is regular $p \in V(p) \subset \mathrm{Cl}(V(p)) \subset U(p)$, where $V(p)$ is an open set, and the collection $\{V(p)\}$ is locally finite. Then for each nonempty $\mathcal{u}(p)$ we construct a nonempty open set as follows:

$$
W(p)=V(p)-U\{\mathrm{Cl}(V(p)) \mid q>p\} .
$$

The $W(p)$ are disjoint and they are open since $\{V(p)\}$ is locally finite. Moreover, the collection $\{W(p)\}$ is locally finite since $\{V(p)\}$ is. $W(p)$ is nonempty since it contains $p$ by (1).

Again using the regularity of $R$, we have $p \in X(p) \subset \mathrm{Cl}(X(p))$ $\subset W(p)$. The collection $\{X(p)\}$ is locally finite and the closures of its elements are disjoint; hence it is a discrete collection, thus by hypothesis countable.

Note. It is not true that in a space satisfying the DCCC every closure preserving open collection is countable. Ceder [4] gives an example of a separable $M_{1}$-space (a space whose basis consists of a countable number of closure preserving open families) which is not second countable.

TheOREM 2.7. For a normal Hausdorff space $R$, the following are equivalent:

(i) $R$ is countably compact.

(ii) Every discrete collection of open subsets of $R$ is finite. 
(iii) Every locally finite collection of open subsets of $R$ is finite.

The equivalence of (i) and (iii) for a normal Hausdorff is known [2, Theorem 4, p. 502].

This theorem gives us another way of seeing that a paracompact, countably compact space is compact.

\section{Images and products.}

THEOREM 3.1. The continuous image of a space satisfying the DCCC satisfies the DCCC.

Proof. The inverse images of the elements of a discrete collection of open sets form a discrete collection.

While the CCC is inherited by open subspaces and the Lindelöf property is inherited by closed subspaces the DCCC is not inherited in general by either open or closed subspaces. Exercise $1 \mathrm{~L}$ from Kelley [6] shows that the property is not inherited by closed subspaces, and the example of Michael referred to above satisfies the DCCC but has an open uncountable subspace with the discrete topology.

We have no example of two spaces satisfying the CCC (DCCC) but whose cartesian product fails to do so. However, we do have a partial result.

Lemma 3.2. In a separable space $R$, every point countable collection of open sets is countable.

Proof. Let $M$ be a countable dense subset of $R$, and let $\mathfrak{u}$ $=\left\{U_{\alpha} \mid \alpha \in a\right\}$ be an open point countable collection. Then for each $U_{\alpha}, M \cap U_{\alpha} \neq \varnothing$. Choose an element $m(\alpha) \in M \cap U_{\alpha}$ for each $U_{\alpha}$. Since $\mathcal{U}$ is point countable, $m(\alpha)$ is in at most countably many sets of $\mathcal{~}$.

Theorem 3.3. If $X$ is separable and $Y$ satisfies the CCC (DCCC), then $X \times Y$ satisfies the CCC $(D C C C)$.

Proof. We prove the theorem for the case in which $Y$ satisfies the CCC. The proof for the other case is similar.

Let $\left\{W_{\alpha} \mid \alpha \in Q\right\}$ be a disjoint collection of open sets in $X \times Y$. Then for $\left(x_{\alpha}, y_{\alpha}\right) \in W_{\alpha}$, there exist sets, $U_{\alpha}$ open in $X, V_{\alpha}$ open in $Y$, such that $\left(x_{\alpha}, y_{\alpha}\right) \in U_{\alpha} \times V_{\alpha} \subset W_{\alpha}$.

Now the family $\left\{U_{\alpha} \mid \alpha \in a\right\}$ is point countable, for consider the collection $\left\{U_{\alpha} \mid x \in U_{\alpha}\right\}$. The corresponding collection of $V_{\alpha}$ 's must be disjoint, for if there were some $y \in V_{\alpha} \cap V_{\beta}$, then $(x, y) \in U_{\alpha} \times V_{\alpha}$ 
$\cap U_{\beta} \times V_{\beta}$. This implies $(x, y) \in W_{\alpha} \cap W_{\beta}$, which is impossible. Therefore, since $Y$ satisfies the CCC, $\left\{U_{\alpha} \mid x \in U_{\alpha}\right\}$ must be countable, and by the lemma $\left\{U_{\alpha} \mid \alpha \in Q\right\}$ is countable.

Now although $W_{\alpha} \neq W_{\beta}, \alpha \neq \beta$, it may happen that $U_{\alpha}=U_{\beta}$, $\alpha \neq \beta$. However by the above argument the set of such indices $\left\{\alpha \mid U_{\alpha} \in \mathcal{u} ; U_{\alpha}=U_{\beta}\right\}$ must be countable. Therefore the fact that $\left\{U_{\alpha} \mid \alpha \in Q\right\}$ is countable implies that $\left\{W_{\alpha} \mid \alpha \in Q\right\}$ must be countable, and $X \times Y$ satisfies the CCC.

\section{REFERENCES}

1. R. Arens and J. Dugundji, Remark on the concept of compactness, Portugal. Math. 9 (1950), 141-143.

2. R. W. Bagley, E. H. Connell and J. D. McKnight, Jr. On properties characterizing pseudo-compact spaces, Proc. Amer. Math. Soc. 9 (1958), 500-506.

3. R. H. Bing, Metrization of topological spaces, Canad. J. Math. 3 (1951), 175-186.

4. J. Ceder, Some generalizations of metric spaces, Pacific J. Math. 11 (1961), $105-125$.

5. C. H. Dowker, On a theorem of Hanner, Ark. Mat. 2 (1952), 307-313.

6. J. L. Kelley, General topology, Van Nostrand, Princeton, N. J., 1955.

7. E. Michael, The product of a normal space and a metric space need not be normal, Bull. Amer. Math. Soc. 69 (1963), 375-376. 833.

8. - - A note on paracompact spaces, Proc. Amer. Math. Soc. 4 (1953), 831-

UNIVERSity OF ST. ThOMAS 